\title{
Scopine isolated in the gas phase
}

Patricia Écija, Monsterrat Vallejo-López, Iciar Uriarte, Francisco J. Basterretxea*, Alberto Lesarri ${ }^{\dagger}$, José A. Fernández, Emilio J. Cocinero*

Departamento de Química Física, Facultad de Ciencia y Tecnología

Universidad del País Vasco (UPV/EHU)

Apartado 644, 48080 Bilbao (Spain)

E-mail: franciscojose.basterretxea@ehu.eus E-mail: emiliojose.cocinero@ehu.es

Homepage: http://www.grupodeespectroscopia.es/MW

†Departamento de Química Física y Química Inorgánica

Facultad de Ciencias, Universidad de Valladolid

47011 Valladolid (Spain)

Supporting information for this article is available on the WWW under http:// $x x x x x x x x x x x$ 


\begin{abstract}
The rotational spectrum of the tropane alkaloid scopine has been detected by Fourier transform microwave spectroscopy in a pulsed supersonic jet. A non-conventional method for bringing the molecules intact into the gas phase was used, mixing scopine syrup with solid-glycine powder and vaporizing the solid mixture with an UV ultrafast laser beam. Laser vaporization prevented the easy isomerization to scopoline previously observed when conventional heating. A single conformer was unambiguously observed in the supersonic jet, which corresponds to the energetically most stable species according to quantum chemistry calculations. Rotational and centrifugal distortion constants have been accurately determined. The spectrum reveals hyperfine splittings due to the combined effect of hindered rotation of the methyl group and the presence of a quadrupolar nucleus $\left({ }^{14} \mathrm{~N}\right)$. This additional information allowed determining the angle of $N$-methyl inversion between the $\mathrm{N}$ $\mathrm{CH}_{3}$ bond and the bicyclic C-N-C plane $\left(135^{\circ}\right)$, as well as the internal rotation barrier of the methyl group $\left(6.24(1) \mathrm{kJ} \mathrm{mol}^{-1}\right)$.
\end{abstract}




\section{INTRODUCTION}

Molecules derived from the tropane bicycle (N-methyl-8-azabicyclo[3.2.1]octane) are broadly used in the pharmaceutical industry. ${ }^{1}$ These compounds find many applications as mydriatics, antiemetics, antispasmodics, anesthetics, and bronchodilators. ${ }^{2,3,4,5}$ Especially important are tropane alkaloids that can be found naturally in many plants of the family Solanaceae, such as atropine and cocaine.

Tropane derivatives having epoxy and hydroxy groups in the 2-carbon bridge are of considerable importance. For example, scopine (6,7-epoxytropine) is an exo-6,7-epoxytropane derivative on which several natural products such as scopolamine are based. Recent interest in 6-hydroxysubstituted tropanes has included valuable natural targets such as schizanthines, Bao Gong Teng derivatives and calystegines. ${ }^{6}$ From the stereochemical point of view, epoxytropanes are interesting molecules, as the influence of the epoxy group can affect reactivity, ring conformation and $\mathrm{N}$ methyl inversion.

In a previous work, our group attempted to detect the rotational spectrum of scopine in the gas phase using microwave spectroscopy in a supersonic jet expansion. ${ }^{7}$ In that experiment, the sample was heated at ca. $90{ }^{\circ} \mathrm{C}$ in the pulsed injector. As a result, the detection of scopine was not possible, since at that temperature the structural isomerization of scopine into scopoline (oscine) by intramolecular cyclation was strongly favored. This behavior was explained taking into account that the three-membered ring epoxide group of scopine is considerably strained, making the molecule more reactive. In this work we report the rotational spectrum of scopine for the first time. For this goal, we have employed an alternative method to bring scopine molecules into the gas phase, consisting in the vaporization of a solid sample of scopine mixed with a glycine matrix using an UV ultrafast laser. This method avoids heating the sample and hence its structural isomerization (see Experimental section). This study follows previous work by our group on stereochemical properties of tropane derivatives by rotational spectroscopy in jet expansions, such as tropinone and its intermolecular complexes. ${ }^{8,9}$ Gas-phase rotational spectroscopy with microwave techniques offers the advantage that molecules are not affected by solvent or crystal packing effects present in condensed media, producing high resolution $(\mathrm{kHz})$ spectra that result in the most accurate structural descriptions for the isolated molecule. This allows describing the conformational landscape of the isolated molecule, which ultimately governs its stereochemical properties. The highly accurate data obtained by microwave techniques can also be used as a benchmark to test the performance of theoretical ab initio quantum chemistry calculations, needed to provide adequate molecular models. 


\section{EXPERIMENTAL AND COMPUTATIONAL SECTION}

The experimental work was carried out in a 4-18 GHz Fourier transform microwave spectrometer (FT-MW) based on the Balle-Flygare design, ${ }^{10}$ constructed at the UPV/EHU and fully described elsewhere. ${ }^{11}$ The commercial samples of scopine (97\%) are syrups and the conventional heating method results in an intramolecular reaction yielding scopoline. So, a non-conventional method of vaporization was designed, where scopine ( 33\%), finely powdered glycine $(\sim 66 \%)$ and a commercial binder (minimum addition) were mixed and mechanically pressed to obtain cylindertype solid samples. These were vaporized by the beam of a picosecond Nd:YAG laser working at third harmonic $(355 \mathrm{~nm})$, giving $\sim 5 \mathrm{~mJ}$ per pulse in a Smalley-type laser ablation source. ${ }^{12}$ The laser vaporization system is described elsewhere. ${ }^{13}$ The vaporized sample was mixed with 4-6 bar of neon carrier gas and adiabatically expanded by $200 \mu$ s duration pulses in a vacuum chamber to achieve low temperatures $(\mathrm{T}<5 \mathrm{~K})$ that effectively depopulate excited ro-vibrational levels. After expansion, molecules in the jet absorb low power (0.1-100 $\mathrm{mW})$ microwave pulses of $1 \mu$ s duration, causing a spontaneous emission that is detected in the time domain and Fourier transformed. The accuracy of the frequency measurements is better than $3 \mathrm{kHz}$, and spectral lines separated less than ca. $10 \mathrm{kHz}$ can be resolved. Because the jet and resonator axis are coaxial, all transitions appear split into two Doppler components.

The experimental work was supported by ab initio (MP2) and DFT methods. All the quantum chemistry calculations were implemented in Gaussian 09. ${ }^{14}$ Becke's B3LYP hybrid functional and Truhlar's dispersion-corrected M06-2X functional ${ }^{15}$ were used in the DFT calculations. The Pople 6-311++G(d,p) basis set was used in all cases. 


\section{RESULTS AND DISCUSSION}

The starting point of the study was the analysis of the molecular potential energy surface (PES). First of all, the 6-membered tropane ring could adopt either chair, higher-energy twisted or even boat conformations. Second, $N$-methyl equatorial/axial isomerism characteristic of tropanes was predicted. Finally, the hydroxyl group can also adopt two different configurations: in one of them (doubled by symmetry) the O-H points outwards the molecule breaking the $C_{S}$ symmetry; in the other configuration the $\mathrm{O}-\mathrm{H}$ is directed inwards the bicycle ring, retaining the $C_{S}$ arrangement in the tropane. So, according to those degrees of freedom, the molecule can adopt a number of different structures. Figure 1 shows the MP2 computed structures of the lowest-lying conformers. Ab initio calculations showed that eight conformers of scopine are expected in the $0-34 \mathrm{~kJ} / \mathrm{mol}$ energy range. ${ }^{7}$ The most stable structure (Conformer CEO) exhibits a chair configuration in the 6-membered ring, an equatorial arrangement of the $N$-methyl substituent isemerism-and the hydroxyl group pointing out of the molecule where steric effects are lower. The next conformer (CEI) is $2.1 \mathrm{~kJ} \mathrm{~mol}^{-1}$ above the lowest-lying one, and differs from it only in the orientation of the $\alpha$-hydroxyl $\mathrm{H}$ atom, that points towards the bicycle ring. Conformer BEO, at $10.3 \mathrm{~kJ} \mathrm{~mol}^{-1}$ over the global energy minimum, is the lowest-lying structure in which the tropane ring adopts a boat-type structure, favored by the byciclic ring. The two lowest energy conformers with an axial arrangement, in which the $\mathrm{CH}_{3}$ internal rotor orients opposite the epoxy group, have substantially high energies (11.6 and $13.4 \mathrm{~kJ}$ $\mathrm{mol}^{-1}$ relative to the lowest-lying species). The rest of the conformers are above $14.0 \mathrm{~kJ} \mathrm{~mol}^{-1}$ from the global energy minimum, and are not expected to cannot be populated in the low temperatures attained in the jet.

Initial scans showed several rotational transitions that could be assigned to the lowest-lying conformer and were used to obtain preliminary rotational constants. These, in turn, allowed to find further transitions and improvement in the rotational parameters. Rotational transitions were assigned in the 8200-12700 MHz range. The list of all assigned transitions $\left(J^{\prime \prime}, K^{\prime \prime}{ }_{-1}, K_{+1} \rightarrow J^{\prime}\right.$, $K_{-1}{ }_{-1}, K^{\prime}{ }_{+1}$ ) are collected in Table S1 (Supplementary Information). An example of the observed transitions is shown in Figure 2. All transitions were of $R$-type $(\Delta J=+1)$ and obeyed $\mu_{\mathrm{a}}$-type selection rules. Each of them appears spread split in a number of components (typically separated less than $0.5 \mathrm{MHz}$ ) due to quadrupole hyperfine coupling-splittings arising from the ${ }^{14} \mathrm{~N}$ nucleus, giving $F^{\prime \prime} \rightarrow F^{\prime}$ transitions (where the $F$ quantum number can take the values $F=J+I, J+I-1, \ldots$, $|J-I|$, and $I$ is the quantum number for nuclear spin: $\left.I\left({ }^{14} \mathrm{~N}\right)=1\right) \cdot{ }^{16}$ Another relevant feature of the spectrum was a small doubling detectable in some transitions (see Figure 1), different from the $\sim 50$ - 
$80 \mathrm{kHz}$ line doubling caused by the instrumental Doppler effect. This tunnelling doubling was attributed to the hindered internal rotation of a single $C_{3}$-symmetric methyl group, splitting the torsional levels into A and E symmetry species. All observed transitions were fitted to a semi-rigid Watson Hamiltonian with centrifugal distortion. ${ }^{17}$ This allowed us to obtain the rotational constants and two of the centrifugal distortion constants $\left(D_{J}\right.$ and $\left.D_{J K}\right)$. The fit also included the diagonal elements of the nuclear quadrupole coupling tensor $(\chi \alpha \alpha, \alpha \alpha=a a, b b, c c)^{18,16}$ and internal rotation terms expressed in the Internal Axis Method (IAM) formalism ${ }^{19}$ of Woods and van Eijck. The internal rotation analysis produces additional structural information, since the experimental splittings depend both on the barrier height and the orientation of the internal $\mathrm{CH}_{3}$ rotor with respect to the principal axes. The XIAM program ${ }^{20}$ developed by Hartwig and Dreizler was used for these fittings. The internal rotation barrier height of the methyl group has been found at $6.24(1) \mathrm{kJ} \mathrm{mol}^{-1}$. All the fitted constants are given Table 1 and compared to ab initio predictions. The predicted constants for the rest of conformers and the computed Cartesian coordinates of the assigned conformer appear in Tables S2 and S3 (Supplementary Information), respectively.

Although the experimental rotational constants do not allow to obtain the full structure of the observed conformer of scopine, the ${ }^{14} \mathrm{~N}$ nuclear quadrupole constants $\chi_{\alpha \alpha}$ exhibit a dependence of the $\mathrm{CH}_{3}$ rotor position with respect to the bicyclic frame. This arises because the $\chi \alpha \alpha$ constants are essentially dependent on the electronic environment around the ${ }^{14} \mathrm{~N}$ nucleus and the orientation of the principal inertial axes. Figure 3 shows the theoretical predictions and experimental values of the nuclear quadrupole coupling tensor diagonal elements for various inversion angles formed by the $\mathrm{N}-\mathrm{CH}_{3}$ bond and the $\mathrm{C}-\mathrm{N}-\mathrm{C}$ plane near the minimum energy structure. The comparison between the experimental and computed plots allows estimating the effective $\mathrm{CH}_{3}$ rotor angle with respect to the bicyclic frame in the $131.8^{\circ}-137.8^{\circ}$ range. This value is consistent with the observations in other tropane-alkaloids, like tropinone ${ }^{8}\left(134.7^{\circ}-135.7^{\circ}\right)$ and scopoline ${ }^{7}\left(134.0^{\circ}-137.5^{\circ}\right)$. The inversion angles corresponding to the computed minimum energy structures also lie within this interval. The molecular energy change with inversion angle is also presented in Figure 3, showing the agreement with $\chi_{\alpha \alpha}$ calculations.

In the observed conformer (CEO), the $\mathrm{H}$ atom in the hydroxyl group points out of the molecule, so this conformer is doubly-degenerated and the dihedral angle $\tau\left(\mathrm{H}-\mathrm{O}_{11}-\mathrm{C}_{3}-\mathrm{H}\right)$ can be ca. $\pm 60^{\circ}$. The next conformer in energy (CEI) is only $2.1 \mathrm{kJmol}^{-1}$ above the lowest-lying one, and differs in the orientation of the $\mathrm{H}$ atom that points towards the bicycle ring. In this case the dihedral angle $\tau(\mathrm{H}-$ $\left.\mathrm{O}_{11}-\mathrm{C}_{3}-\mathrm{H}\right)$ is $180^{\circ}$, giving a $C_{S}$ molecular symmetry. The non-observation of this conformer could be explained by a conformational relaxation in the jet, as both CEO and CEI conformers are connected by a small barrier of $4.6 \mathrm{~kJ} \mathrm{~mol}^{-1}$ (see Figure 4, top panel). Previous studies suggested 
that barriers in the $5-12 \mathrm{~kJ} \mathrm{~mol}^{-1}$ range could prevent the observation of high energy conformers.

The $N$-methyl group of conformer CEO adopts an equatorial position relative to the tropane ring. The corresponding axial conformer CAO lies about $13.4 \mathrm{~kJ} \mathrm{~mol}^{-1}$ higher and has not been observed due to the low population attained in the jet. The energy barrier for the $\mathrm{CAO} \rightarrow \mathrm{CEO}$ conversion has been calculated (DFT) to be $22.6 \mathrm{~kJ} \mathrm{~mol}^{-1}$ (Figure 4, bottom panel). The equatorial conformer CEO might be partially stabilized by the presence of a weak hydrogen bond with the epoxy group (C$\mathrm{H} \cdots \mathrm{O}$ ). The stabilization energy of this bond can be estimated from data of other tropane alkaloids also studied by rotational spectroscopy (Figure 5). In tropinone ${ }^{8}$, both equatorial and axial conformers were observed, with an equatorial/axial population ratio in the jet near 2/1, corresponding to a relative energy of ca. $2 \mathrm{~kJ} \mathrm{~mol}^{-1}$ at $373 \mathrm{~K}$, and an $a b$ initio calculated inversion barrier of $40 \mathrm{~kJ} \mathrm{~mol}^{-1}$. Assuming that the energy of $N$-methyl axial/equatorial inversion in tropinone is the same for scopine, and that the energies of $N$-methyl inversion and hydrogen bond are simply additive, the stabilization energy of the weak $\mathrm{C}-\mathrm{H} \cdots \mathrm{O}$ interaction in scopine can be estimated to be about $11 \mathrm{~kJ} / \mathrm{mol}^{-1}$. On the other side, in scopoline ${ }^{7}$, the intramolecular $\mathrm{O}-\mathrm{H} \cdots \mathrm{N}$ hydrogen bond forces the $\mathrm{N}$-methyl group to the less stable axial form, so it cannot be compared directly with tropinone and scopine. 


\section{CONCLUSIONS}

In conclusion, in this work we have detected the scopine molecule in the gas phase for the first time. The most stable conformer of scopine has been observed by rotational spectroscopy, leading to accurate determination of rotational and centrifugal distortion constants. Internal torsional splittings and quadrupole hyperfine structure present in the spectra has allowed determination of the methyl rotor angle with respect to the bicyclo frame and the internal rotor barrier height. Using ultrafast laser vaporization has been crucial as the samples react at moderately low temperatures, preventing the use of heating techniques for vaporization. No spectral lines coming from the reaction product of scopoline have been detected, demonstrating that the laser energy transfer to the matrix is relatively clean and that it does not decompose or transform the sample appreciably. A new method of sample preparation where the syrup target was mixed with a solid to obtain a solid sample vaporizable by a laser pulse could be a good alternative to study syrups in gas phase, gaining access to samples previously unobservable or where where traditional heating methods lead to chemical decomposition or other reactions.

\section{ACKNOWLEDGMENTS}

Financial support from the MICINN and MINECO (CTQ-2014-54464-R, CTQ-2015-68148), the Basque Government (Consolidated Groups, IT520-10) and the UPV/EHU (UFI11/23) is gratefully acknowledged. E.J.C, M.V.-L. and I.U. acknowledges also a "Ramón y Cajal", "Juan de la Cierva" and FPU contracts from the MINECO. Computational resources and laser facilities of the UPV/EHU were used in this work (SGIker and I2Basque) and European funding (ERDF and ESF). 
Figure 1. MP2 computed structures of the eight lowest-lying conformers of scopine. We used the notation Chair/Boat (C/B), Equatorial/Axial (E/A) and Inwards/Outwards (I/O) to name the different conformers. Relative energies to the global minimum are indicated, values in parentheses corresponding to Gibbs free energies All the $\left(\mathrm{kJ} \mathrm{mol}^{-1}\right)$.

CEO
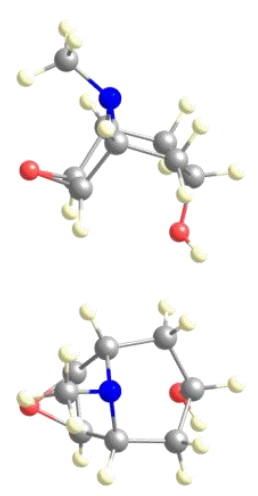

$0.0(0.0)$

CAO
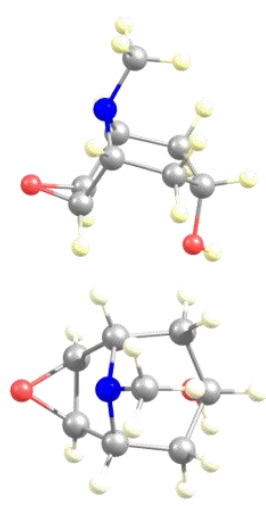

$13.4(12.6)$
CEI
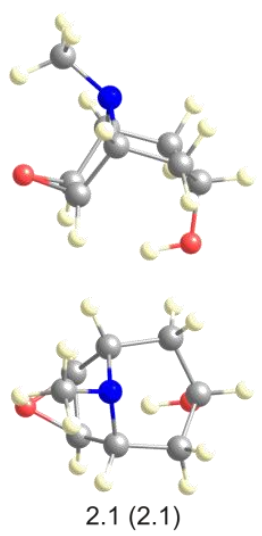

BEI
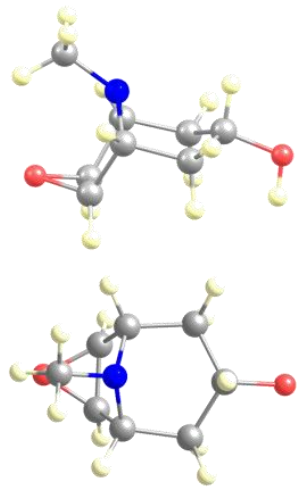

$14.7(14.1)$
BEO
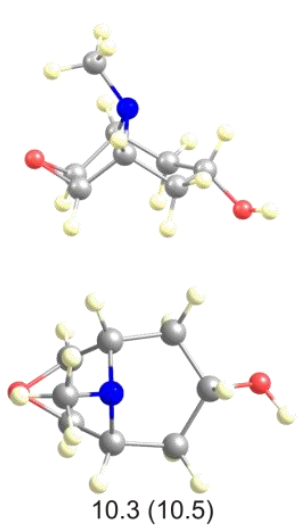

BAO
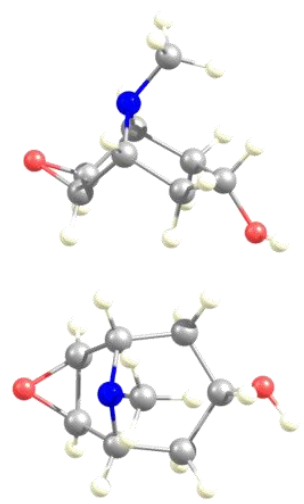

32.5 (29.4)

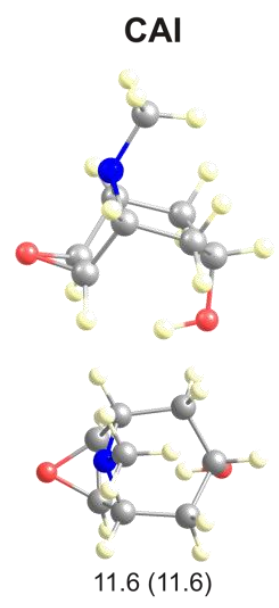

BAI
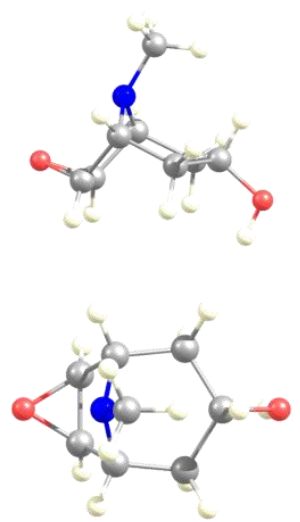

34.0 (32.3) 
Figure 2. Part of the rotational spectrum of scopine showing the $J\left(K_{-1}, K_{+1}\right)=4(2,3) \leftarrow 3(2,2)$ transition of the CEO conformer. The transition splits in several components due to nuclear quadrupole coupling interaction, of which the $F=5 \leftarrow 4$ and $4 \leftarrow 3$ components are shown. Additionally, each component appears split in A and E lines due to hindered internal rotation of the methyl internal rotor. All lines are finally doubled by the instrumental Doppler effect.

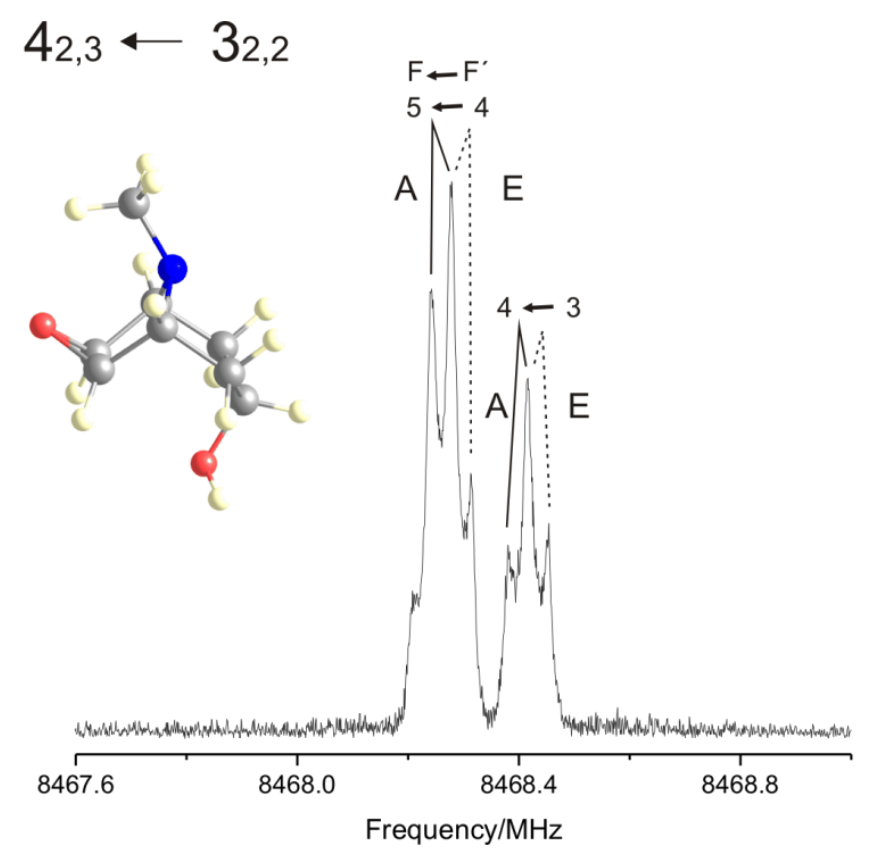


Figure 3. Comparison between experimental and calculated values of the nuclear quadrupole coupling tensor diagonal elements $\chi_{a a}, \chi_{b b}$, and $\chi_{c c}$ for different values of the inversion angle (defined as the angle between the $\mathrm{N}-\mathrm{CH}_{3}$ bond and the $\mathrm{C}-\mathrm{N}-\mathrm{C}$ plane). The grey-shaded interval between $131.8^{\circ}$ and $137.8^{\circ}$ gives the confidence interval for which the inversion angle can be specified, taking into account the experimental errors in the $\chi_{\alpha \alpha}$ diagonal elements. The inversion angles corresponding to the minimum energy structures, as given by quantum chemical calculations, all lie within the proposed confidence interval. Also shown are the MP2 energy change with the inversion angle and the predicted structure of the molecule.

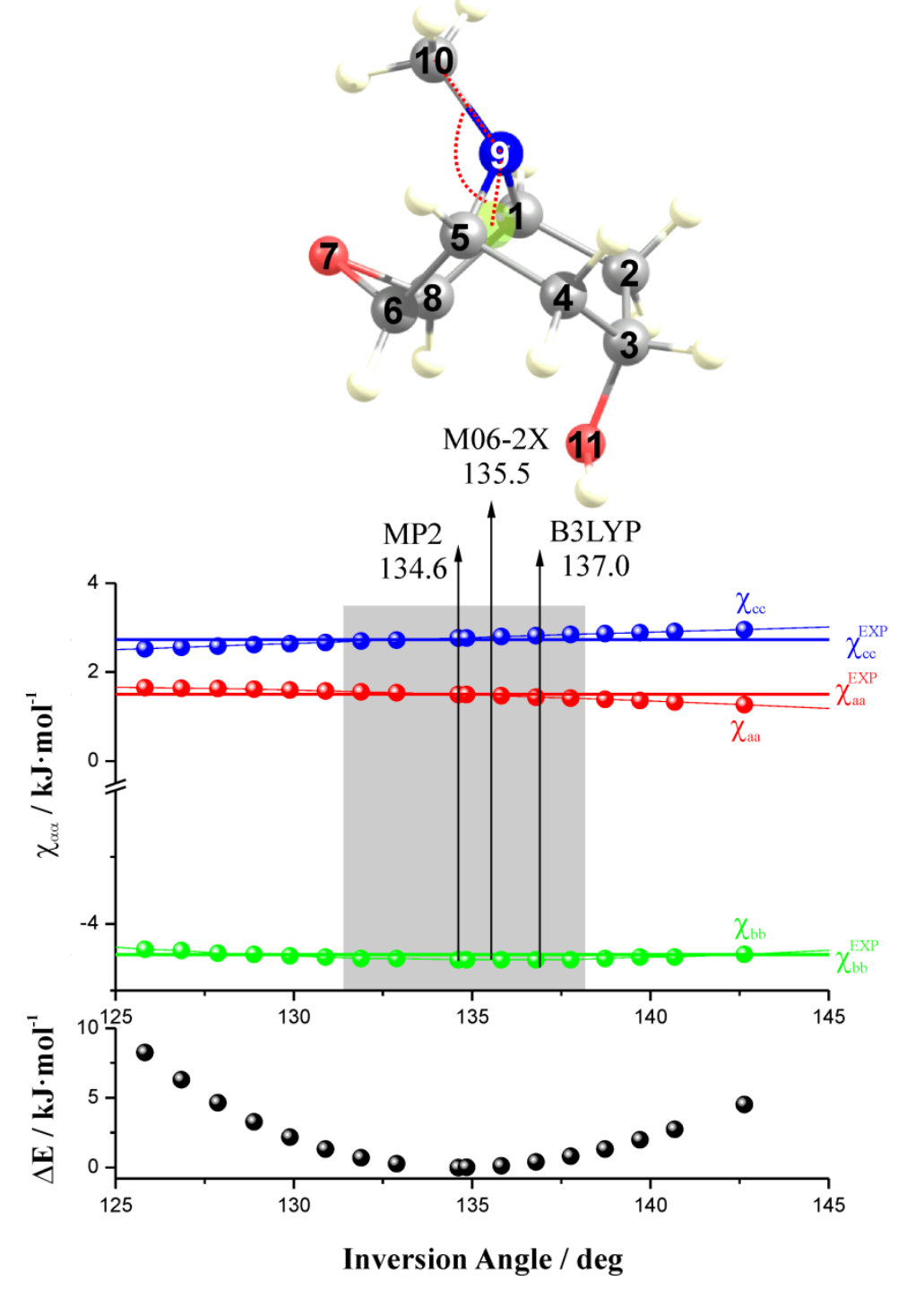


Figure 4. Profiles of the potential energy (M06-2X) showing barriers for the interconversion between the two lowest-lying energy conformers (CEO and CEI, top panel) and $N$-methyl inversion (CEO and CAO, bottom panel).
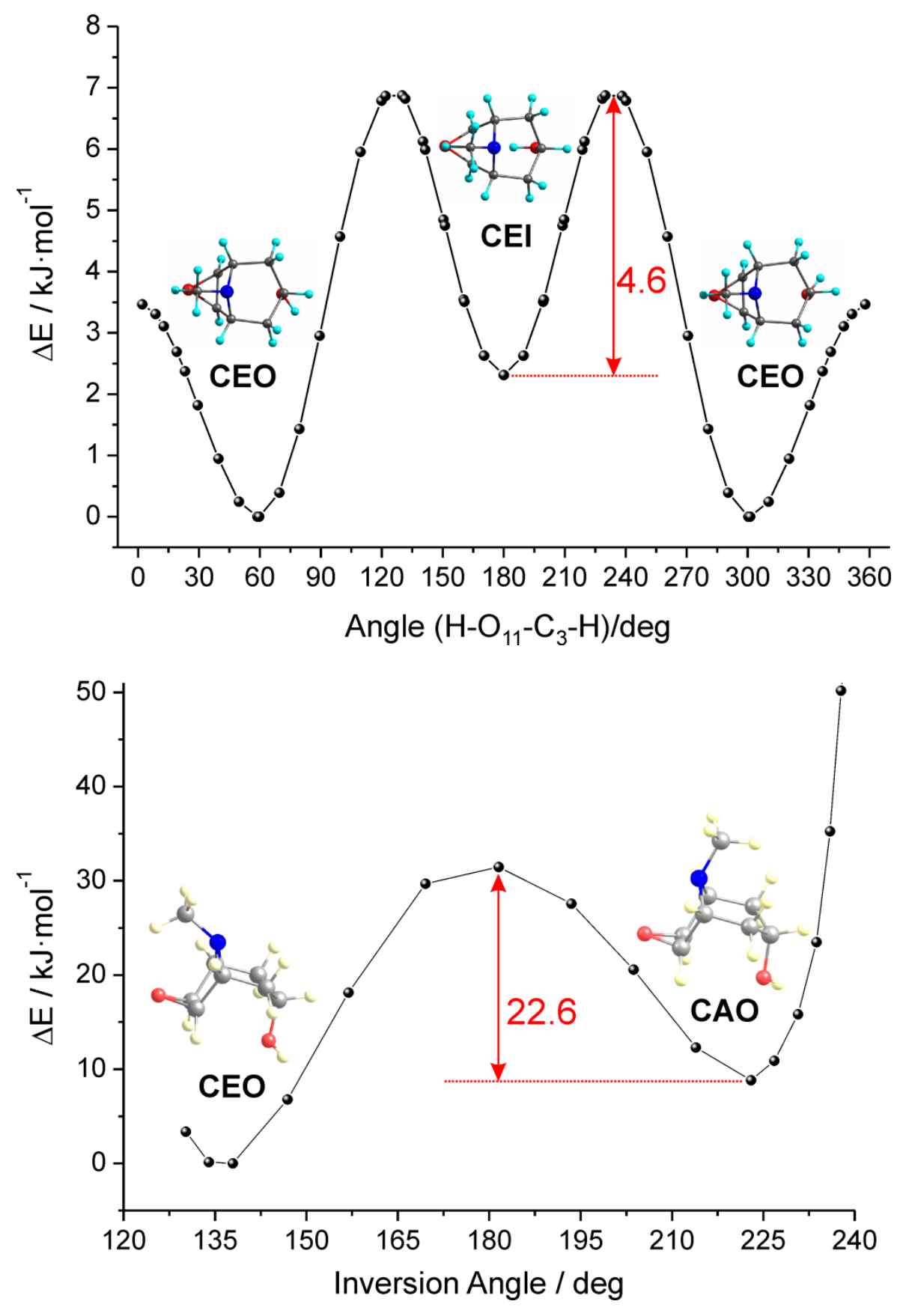
Figure 5. MP2 predicted relative energies $\left(\mathrm{kJ} \mathrm{mol}^{-1}\right)$ in equatorial/axial conformers of several alkaloids presenting $N$-methyl inversion. The red cross means that the conformer was not observed by rotational spectroscopy.

Tropinone

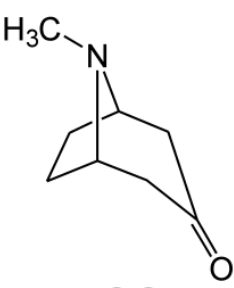

0.0

Scopoline

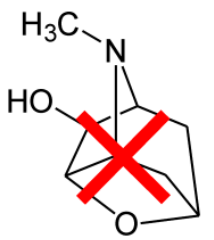

16.8

Scopine<smiles>CN1[C@H]2CC[C@@H]1CC(=O)C2</smiles>

2.3

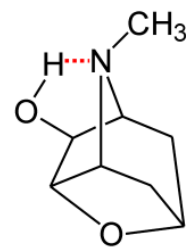

0.0

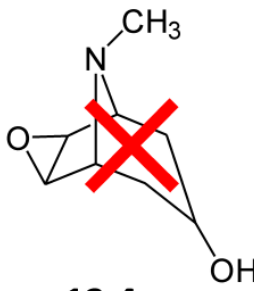

13.4 
Table 1. Experimentally fitted and calculated parameters for the detected conformer of scopine.

\begin{tabular}{|c|c|c|c|c|}
\hline & Experiment & MP2 & B3LYP & M06-2X \\
\hline $\mathrm{A} / \mathrm{MHz}^{[\mathrm{a}]}$ & $1866.6159(57)^{[\mathrm{b}]}$ & 1866.1 & 1854.0 & 1873.5 \\
\hline $\mathrm{B} / \mathrm{MHz}$ & $1110.10005(14)$ & 1117.3 & 1094.3 & 1118.5 \\
\hline $\mathrm{C} / \mathrm{MHz}$ & $1008.87127(17)$ & 1014.3 & 994.5 & 1016.5 \\
\hline $\mathrm{D}_{\mathrm{J}} / \mathrm{kHz}$ & $0.0421(25)$ & 0.047 & 0.056 & 0.049 \\
\hline $\mathrm{D}_{\mathrm{JK}} / \mathrm{kHz}$ & $-0.124(31)$ & -0.009 & -0.031 & -0.016 \\
\hline $\mathrm{D}_{\mathrm{K}} / \mathrm{kHz}$ & {$[0.0]$} & 0.082 & 0.109 & 0.084 \\
\hline $\mathrm{d}_{1} / \mathrm{Hz}$ & {$[0.0]$} & -6.17 & -8.11 & -6.67 \\
\hline $\mathrm{d}_{2} / \mathrm{Hz}$ & {$[0.0]$} & -0.11 & -0.27 & -0.16 \\
\hline$\chi_{\mathrm{aa}} / \mathrm{MHz}$ & $1.5133(99)$ & 1.50 & 1.51 & 1.50 \\
\hline$\chi_{\mathrm{bb}} / \mathrm{MHz}$ & $-4.233(12)$ & -4.27 & -4.59 & -4.27 \\
\hline$\chi_{\mathrm{cc}} / \mathrm{MHz}$ & $2.720(12)$ & 2.77 & 3.07 & 2.77 \\
\hline$\left|\mu_{\mathrm{a}}\right| / \mathrm{D}$ & & 1.82 & 1.81 & 1.86 \\
\hline$\left|\mu_{\mathrm{b}}\right| / \mathrm{D}$ & & 1.32 & 1.44 & 1.34 \\
\hline $\mid \begin{array}{l}\mu_{\mathrm{c}} \mid / \mathrm{D} \\
\mu_{\mathrm{TOT}} / \mathrm{D}\end{array}$ & & 1.06 & 1.05 & 1.07 \\
\hline$\angle(\mathrm{a}, \mathrm{i}) / \mathrm{deg}^{[\mathrm{c}]}$ & $174.01^{[\mathrm{d}]}$ & 174.01 & 173.73 & 171.73 \\
\hline$\angle(b, \mathrm{i}) / \mathrm{deg}$ & $95.98[\mathrm{~d}]$ & 95.98 & 96.26 & 97.94 \\
\hline$\angle(\mathrm{c}, \mathrm{i}) / \mathrm{deg}$ & $90.35^{[\mathrm{d}]}$ & 90.35 & 90.31 & 92.29 \\
\hline $\mathrm{I}_{\alpha} / \mathrm{u} \AA^{2}$ & $3.275^{[\mathrm{d}]}$ & 3.275 & 3.251 & 3.250 \\
\hline $\mathrm{V}_{3} / \mathrm{kJ} \mathrm{mol}^{-1}[\mathrm{e}]$ & $6.24(1)$ & 5.99 & 5.83 & 6.86 \\
\hline$N^{[f]}$ & 66 & & & \\
\hline$\sigma / \mathrm{kHz}$ & 2.7 & & & \\
\hline
\end{tabular}

[a] Rotational constants (A, B, C); centrifugal distortion constants (DJ, DIK, $D_{K}, d_{1}, d_{2}$ ); nuclear quadrupole coupling elements $\left(\chi_{\mathrm{aa}}, \chi_{\mathrm{bb}}, \chi_{\mathrm{cc}}\right)$ and dipole moment components $\left(\mu_{\mathrm{a}}, \mu_{\mathrm{b}}, \mu_{\mathrm{c}}, 1 \mathrm{D} \approx 3.336 \times 10^{-30} \mathrm{C} \mathrm{m}\right.$ ). [b] Standard errors in parentheses in units of the last digit. [c] $\angle$ (g,i): Angles defining the orientation of the internal rotor axis (i) with respect to the principal inertial axes ( $\mathrm{g}=\mathrm{a}, \mathrm{b}$, c); moment of inertia $\left(I_{\alpha}\right)$ of the internal rotor with respect to its $C_{3}$ axis. [d] This value cannot be experimentally determined and has been fixed in the fit to the MP2-calculated value. [e] Three-fold barrier height. [f] Number of transitions (N) and rms deviation $(\sigma)$ of the fit. 


\section{SUPPLEMENTARY INFORMATION}

Table S1. Rotational transitions ( $\mu_{\mathrm{a}}$-type, Obs.) and residuals (o.-c.) of scopine (all frequencies in $\mathrm{MHz}$ ).

\begin{tabular}{|c|c|c|c|c|c|c|c|c|c|c|}
\hline $\mathrm{J}^{\prime}$ & $\mathrm{K}^{\prime}-1$ & $\mathrm{~K}^{\prime}+1$ & $\mathrm{~J}^{\prime \prime}$ & $K^{\prime \prime}-1$ & $\mathrm{~K}^{\prime \prime}+1$ & $\mathbf{F}^{\prime}$ & $\mathbf{F}^{\prime \prime}$ & Sym & Obs.a & $0 .-c \cdot b$ \\
\hline \multirow[t]{4}{*}{4} & 1 & 4 & 3 & 1 & 3 & 5 & 4 & $A$ & 8256.017 & -0.000 \\
\hline & & & & & & 5 & 4 & $\mathrm{E}$ & 8256.017 & -0.000 \\
\hline & & & & & & 4 & 3 & A & 8255.987 & -0.002 \\
\hline & & & & & & 4 & 3 & $\mathrm{E}$ & 8255.987 & -0.002 \\
\hline \multirow[t]{6}{*}{4} & 0 & 4 & 3 & 0 & 3 & 5 & 4 & $\mathrm{~A}$ & 8385.642 & -0.000 \\
\hline & & & & & & 5 & 4 & $\mathrm{E}$ & 8385.642 & 0.002 \\
\hline & & & & & & 4 & 3 & $\mathrm{~A}$ & 8385.383 & -0.000 \\
\hline & & & & & & 4 & 3 & $\mathrm{E}$ & 8385.383 & 0.002 \\
\hline & & & & & & 3 & 2 & $\mathrm{~A}$ & 8385.718 & -0.004 \\
\hline & & & & & & 3 & 2 & $\mathrm{E}$ & 8385.718 & -0.002 \\
\hline \multirow[t]{4}{*}{4} & 2 & 3 & 3 & 2 & 2 & 5 & 4 & A & 8468.430 & 0.005 \\
\hline & & & & & & 5 & 4 & $\mathrm{E}$ & 8468.471 & 0.002 \\
\hline & & & & & & 4 & 3 & $\mathrm{~A}$ & 8468.598 & 0.002 \\
\hline & & & & & & 4 & 3 & $\mathrm{E}$ & 8468.643 & 0.003 \\
\hline \multirow[t]{4}{*}{4} & 2 & 2 & 3 & 2 & 1 & 5 & 4 & $\mathrm{~A}$ & 8558.615 & -0.004 \\
\hline & & & & & & 5 & 4 & $\mathrm{E}$ & 8558.578 & 0.002 \\
\hline & & & & & & 4 & 3 & A & 8559.057 & -0.003 \\
\hline & & & & & & 4 & 3 & $\mathrm{E}$ & 8559.020 & 0.002 \\
\hline \multirow[t]{6}{*}{4} & 1 & 3 & 3 & 1 & 2 & 5 & 4 & $\mathrm{~A}$ & 8657.546 & 0.001 \\
\hline & & & & & & 5 & 4 & $\mathrm{E}$ & 8657.546 & 0.003 \\
\hline & & & & & & 4 & 3 & $\mathrm{~A}$ & 8657.546 & -0.001 \\
\hline & & & & & & 4 & 3 & $\mathrm{E}$ & 8657.546 & 0.001 \\
\hline & & & & & & 3 & 2 & A & 8657.682 & -0.001 \\
\hline & & & & & & 3 & 2 & $\mathrm{E}$ & 8657.682 & 0.001 \\
\hline \multirow[t]{4}{*}{5} & 1 & 5 & 4 & 1 & 4 & 6 & 5 & $\mathrm{~A}$ & 10305.692 & 0.000 \\
\hline & & & & & & 6 & 5 & $\mathrm{E}$ & 10305.692 & 0.001 \\
\hline & & & & & & 5 & 4 & $\mathrm{~A}$ & 10305.631 & -0.001 \\
\hline & & & & & & 5 & 4 & $\mathrm{E}$ & 10305.631 & -0.000 \\
\hline \multirow[t]{6}{*}{5} & 0 & 5 & 4 & 0 & 4 & 6 & 5 & A & 10425.936 & -0.002 \\
\hline & & & & & & 6 & 5 & $\mathrm{E}$ & 10425.936 & 0.001 \\
\hline & & & & & & 5 & 4 & $A$ & 10425.659 & -0.002 \\
\hline & & & & & & 5 & 4 & $\mathrm{E}$ & 10425.659 & 0.001 \\
\hline & & & & & & 4 & 3 & A & 10425.984 & -0.004 \\
\hline & & & & & & 4 & 3 & $\mathrm{E}$ & 10425.984 & -0.000 \\
\hline \multirow[t]{4}{*}{5} & 2 & 4 & 4 & 2 & 3 & 6 & 5 & A & 10573.727 & -0.007 \\
\hline & & & & & & 6 & 5 & $\mathrm{E}$ & 10573.743 & -0.003 \\
\hline & & & & & & 5 & 4 & A & 10573.786 & 0.001 \\
\hline & & & & & & 5 & 4 & $\mathrm{E}$ & 10573.801 & 0.004 \\
\hline \multirow[t]{4}{*}{5} & 2 & 3 & 4 & 2 & 2 & 6 & 5 & A & 10742.410 & 0.003 \\
\hline & & & & & & 6 & 5 & $\mathrm{E}$ & 10742.398 & 0.003 \\
\hline & & & & & & 5 & 4 & $\mathrm{~A}$ & 10742.742 & 0.002 \\
\hline & & & & & & 5 & 4 & $\mathrm{E}$ & 10742.729 & -0.002 \\
\hline \multirow[t]{6}{*}{5} & 1 & 4 & 4 & 1 & 3 & 6 & 5 & A & 10800.269 & 0.000 \\
\hline & & & & & & 6 & 5 & $\mathrm{E}$ & 10800.269 & 0.002 \\
\hline & & & & & & 5 & 4 & $\mathrm{~A}$ & 10800.196 & -0.003 \\
\hline & & & & & & 5 & 4 & $\mathrm{E}$ & 10800.196 & -0.001 \\
\hline & & & & & & 4 & 3 & $\mathrm{~A}$ & 10800.360 & -0.003 \\
\hline & & & & & & 4 & 3 & $\mathrm{E}$ & 10800.360 & -0.001 \\
\hline \multirow[t]{3}{*}{6} & 1 & 6 & 5 & 1 & 5 & 7 & 6 & A & 12348.315 & 0.001 \\
\hline & & & & & & 7 & 6 & $\mathrm{E}$ & 12348.315 & 0.002 \\
\hline & & & & & & 6 & 5 & $A$ & 12348.243 & 0.001 \\
\hline
\end{tabular}




\begin{tabular}{|c|c|c|c|c|c|c|c|c|c|c|}
\hline & & & & & & 6 & 5 & $E$ & 12348.243 & 0.002 \\
\hline & & & & & & 5 & 4 & A & 12348.291 & -0.004 \\
\hline & & & & & & 5 & 4 & $E$ & 12348.291 & -0.003 \\
\hline \multirow[t]{6}{*}{6} & 0 & 6 & 5 & 0 & 5 & 7 & 6 & A & 12446.375 & -0.002 \\
\hline & & & & & & 7 & 6 & $E$ & 12446.375 & 0.002 \\
\hline & & & & & & 6 & 5 & A & 12446.122 & -0.002 \\
\hline & & & & & & 6 & 5 & $E$ & 12446.122 & 0.002 \\
\hline & & & & & & 5 & 4 & A & 12446.411 & 0.004 \\
\hline & & & & & & 5 & 4 & $E$ & 12446.411 & 0.008 \\
\hline \multirow[t]{6}{*}{6} & 2 & 5 & 5 & 2 & 4 & 7 & 6 & A & 12671.329 & 0.002 \\
\hline & & & & & & 7 & 6 & $E$ & 12671.329 & -0.002 \\
\hline & & & & & & 6 & 5 & A & 12671.316 & -0.002 \\
\hline & & & & & & 6 & 5 & $\mathrm{E}$ & 12671.316 & -0.006 \\
\hline & & & & & & 5 & 4 & A & 12671.339 & 0.002 \\
\hline & & & & & & 5 & 4 & $E$ & 12671.339 & -0.001 \\
\hline
\end{tabular}

a Observed.

b Observed minus calculated. 
Table S2. Rotational parameters predicted for the most stable conformers of scopine using MP2 and DFT theory (B3LYP and M06-2X functionals).

\begin{tabular}{|c|c|c|c|c|c|c|c|c|}
\hline & $\begin{array}{c}\text { Conf. CEO } \\
\text { MP2/M062X/B3LYP }\end{array}$ & $\begin{array}{c}\text { Conf. CEI } \\
\text { MP2/M062X/B3LYP }\end{array}$ & $\begin{array}{c}\text { Conf. BEO } \\
\text { MP2/M062X/B3LYP }\end{array}$ & $\begin{array}{c}\text { Conf. CAl } \\
\text { MP2/M062X/B3LYP }\end{array}$ & $\begin{array}{c}\text { Conf. CAO } \\
\text { MP2/M062X/B3LYP }\end{array}$ & $\begin{array}{c}\text { Conf. BEI } \\
\text { MP2/M062X/B3LYP }\end{array}$ & $\begin{array}{c}\text { Conf. BAO* } \\
\text { MP2/M062X/B3LYP }\end{array}$ & $\begin{array}{c}\text { Conf. BAl } \\
\text { MP2/M062X/B3LYP }\end{array}$ \\
\hline $\mathrm{A} / \mathrm{MHz}[\mathrm{a}]$ & $1866 / 1854 / 1874$ & 1869/1876/1856 & 2028/2033/2002 & $1521 / 1526 / 1520$ & $1519 / 1524 / 1519$ & 2029/2032/2002 & 1722/--/1720 & $1759 / 1748 / 1744$ \\
\hline $\mathrm{B} / \mathrm{MHz}$ & $1117 / 1094 / 1119$ & 1104/1101/1084 & $931 / 933 / 924$ & $1303 / 1304 / 1270$ & $1319 / 1320 / 1281$ & $927 / 931 / 921$ & 1049/--/1029 & 1023/1034/1013 \\
\hline $\mathrm{C} / \mathrm{MHz}$ & $1014 / 995 / 1017$ & $1004 / 1003 / 986$ & $888 / 891 / 880$ & $1023 / 1025 / 1003$ & $1032 / 1035 / 1011$ & $884 / 887 / 876$ & 923/--/909 & 909/916/900 \\
\hline $\mathrm{D}_{\mathrm{J}} / \mathrm{kHz}$ & $0.047 / 0.056 / 0.049$ & $0.043 / 0.047 / 0.047$ & $0.020 / 0.020 / 0.021$ & $0.061 / 0.062 / 0.069$ & $0.065 / 0.063 / 0.071$ & 0.019/0.019/0.020 & 0.181/--/0.063 & $0.045 / 0.083 / 0.042$ \\
\hline $\mathrm{D}_{\mathrm{JK}} / \mathrm{kHz}$ & $-0.009 /-0.031 /-0.016$ & $-0.009 /-0.013 /-0.019$ & $0.080 / 0.081 / 0.092$ & $0.038 / 0.085 / 0.031$ & $0.045 / 0.042 / 0.017$ & 0.078/0.079/0.087 & $-0.809 /--/-0.149$ & $-0.073 /-0.301 /-0.038$ \\
\hline $\mathrm{D}_{\mathrm{K}} / \mathrm{kHz}$ & $0.082 / 0.109 / 0.084$ & $0.087 / 0.090 / 0.11$ & $0.11 / 0.098 / 0.12$ & $-0.031 /-0.081 /-0.028$ & $-0.043 /-0.037 /-0.018$ & $0.11 / 0.10 / 0.12$ & 1.267/--/0.372 & 0. $251 / 0.599 / 0.226$ \\
\hline $\mathrm{d}_{1} / \mathrm{Hz}$ & $-6.17 /-8.11 /-6.67$ & $-5.44 /-6.00 /-6.34$ & $-1.08 /-1.03 /-1.39$ & $-16.9 /-17.7 /-19.7$ & $-18.6 /-17.8 /-20.5$ & $-1.08 /-1.02 /-1.36$ & $-49.24 /--/-15.33$ & $-8.80 /-19.74 /-8.80$ \\
\hline $\mathrm{d}_{2} / \mathrm{Hz}$ & $-0.11 /-0.27 /-0.16$ & $-0.11 /-0.18 /-0.22$ & $0.11 / 0.11 /-0.02$ & $-3.83 /-5.40 /-4.06$ & $-4.28 /-4.05 /-3.75$ & $0.09 / 0.08 /-0.02$ & $-0.829 /--/-0.536$ & $0.13 / 0.11 / 0.26$ \\
\hline$\chi_{\mathrm{aa}} / \mathrm{MHz}$ & $1.50 / 1.51 / 1.50$ & $1.49 / 1.57 / 1.51$ & $-1.98 /-2.16 /-2.26$ & $-4.70 /-5.00 /-4.95$ & $-4.71 /-4.91 /-4.99$ & $-2.14 /-2.31 /-2.44$ & $-5.91 /--/-6.66$ & $-6.03 /-6.38 /-6.71$ \\
\hline$\chi_{\mathrm{bb}} / \mathrm{MHz}$ & $-4.27 /-4.59 /-4.27$ & $-4.27 /-4.57 /-4.60$ & $-0.87 /-0.92 /-0.91$ & $1.84 / 1.94 / 1.77$ & $1.87 / 1.88 / 1.83$ & $-0.73 /-0.79 /-0.74$ & 2.98/--/3.40 & $3.03 / 3.20 / 3.41$ \\
\hline$\chi_{\mathrm{CC}} / \mathrm{MHz}$ & $2.77 / 3.07 / 2.77$ & $2.78 / 3.00 / 3.09$ & $2.86 / 3.08 / 3.16$ & 2.86/3.06/-3.18 & $2.84 / 3.03 / 3.15$ & $2.87 / 3.10 / 3.18$ & 2.93/--/3.26 & $3.00 / 3.19 / 3.31$ \\
\hline$\left|\mu_{a}\right| / D$ & $1.8 / 1.9 / 1.8$ & $0.6 / 0.5 / 0.5$ & $0.7 / 0.77 / 0.9$ & $0.3 / 0.3 / 0.3$ & $2.5 / 2.5 / 2.4$ & $0.3 / 0.2 / 0.1$ & $2.8 /--/ 2.3$ & $1.2 / 0.9 / 0.8$ \\
\hline$\left|\mu_{b}\right| / D$ & 1.3/1.3/1.4 & $0.7 / 0.6 / 0.7$ & $0.7 / 0.7 / 0.8$ & $1.8 / 1.7 / 1.7$ & $2.9 / 2.8 / 2.7$ & $1.2 / 1.3 / 1.1$ & $1.2 /--/ 1.1$ & $0.6 / 0.5 / 0.5$ \\
\hline$\left|\mu_{c}\right| / D$ & 1.1/1.1/1.1 & $0.0 / 0.0 / 0.0$ & 1.1/1.1/1.0 & $0.0 / 0.0 / 0.0$ & 1.1/1.1/1.1 & $0.0 / 0.0 / 0.0$ & $1.2 /--/ 1.1$ & $0.0 / 0.0 / 0.0$ \\
\hline$\left|\mu_{\text {TOT }}\right| /$ D & $2.5 / 2.5 / 2.5$ & 0.9/0.8/0.8 & $1.5 / 1.5 / 1.6$ & $1.8 / 1.8 / 1.7$ & $3.9 / 3.9 / 3.8$ & 1.3/1.3/1.2 & $3.3 /--/ 2.8$ & $1.3 / 1.0 / 1.0$ \\
\hline$\Delta(\mathrm{E}+\mathrm{ZPE}) / \mathrm{kJ} \mathrm{mol}^{-1}$ & $0.0 / 0.0 / 0.0$ & $4.7 / 2.1 / 4.3$ & 13.1/10.3/ & $14.1 / 11.6 /$ & 15.6/13.4/ & $15.8 / 14.7 \mid$ & $32.5 /--/ 25.1$ & $34.0 / 29.6 / 26.3$ \\
\hline$\Delta \mathrm{G} / \mathrm{kJ} \mathrm{mol}^{-1}$ & $0.0 / 0.0 / 0.0$ & $5.1 / 2.1 / 5.8$ & $13.5 / 10.5 /$ & 13.1/11.6/ & $14.9 / 12.6 /$ & 17.3/14.1/ & 29.4/--/25.0 & $32.3 / 26.0 / 25.0$ \\
\hline
\end{tabular}

* Conformer BAO M06-2X calculations converge to Conformer CAO structure 
Table S3. Computed cartesian coordinates (in $\AA$ ) for the observed conformer of scopine. A pdf file showing a $3 \mathrm{D}$ view of the molecule has been added. Double-click inside the red rectangle to activate.

\begin{tabular}{|c|c|c|c|c|c|c|c|c|c|}
\hline & \multicolumn{3}{|c|}{ MP2 } & \multicolumn{3}{|c|}{ M06-2X } & \multicolumn{3}{|c|}{ B3LYP } \\
\hline & $x$ & $y$ & Z & $x$ & $y$ & z & $x$ & $\mathrm{y}$ & z \\
\hline C1 & 0.419 & -0.237 & -1.147 & 0.421 & -0.238 & -1.146 & 0.422 & -0.225 & -1.150 \\
\hline C2 & -1.006 & -0.792 & -1.293 & -1.003 & -0.792 & -1.292 & -1.013 & -0.767 & -1.300 \\
\hline C3 & -1.832 & -0.618 & -0.008 & -1.833 & -0.619 & -0.008 & -1.847 & -0.622 & -0.009 \\
\hline C4 & -1.013 & -0.778 & 1.291 & -1.011 & -0.778 & 1.290 & -1.019 & -0.760 & 1.296 \\
\hline C5 & 0.414 & -0.224 & 1.153 & 0.414 & -0.224 & 1.151 & 0.417 & -0.216 & 1.154 \\
\hline C6 & 0.343 & 1.237 & 0.726 & 0.347 & 1.241 & 0.723 & 0.374 & 1.255 & 0.726 \\
\hline 07 & 1.502 & 1.690 & -0.007 & 1.486 & 1.682 & -0.006 & 1.539 & 1.683 & -0.002 \\
\hline C8 & 0.342 & 1.228 & -0.737 & 0.349 & 1.232 & -0.737 & 0.378 & 1.249 & -0.735 \\
\hline N9 & 1.010 & -0.933 & 0.009 & 1.017 & -0.922 & 0.009 & 1.012 & -0.925 & 0.007 \\
\hline C10 & 2.470 & -1.045 & 0.014 & 2.471 & -1.041 & 0.015 & 2.464 & -1.099 & 0.012 \\
\hline 011 & -2.438 & 0.682 & -0.089 & -2.441 & 0.672 & -0.087 & -2.504 & 0.659 & -0.086 \\
\hline H12 & 1.015 & -0.419 & -2.048 & 1.012 & -0.422 & -2.045 & 1.008 & -0.413 & -2.053 \\
\hline H13 & -0.916 & -1.859 & -1.522 & -0.918 & -1.855 & -1.524 & -0.936 & -1.826 & -1.56 \\
\hline H14 & -1.554 & -0.306 & -2.108 & -1.548 & -0.304 & -2.105 & -1.556 & -0.263 & -2.105 \\
\hline H15 & -2.629 & -1.374 & -0.008 & -2.626 & -1.375 & -0.009 & -2.618 & -1.4014 & -0.010 \\
\hline H16 & -1.555 & -0.289 & 2.113 & -1.551 & -0.291 & 2.111 & -1.558 & -0.262 & 2.111 \\
\hline H17 & -0.927 & -1.842 & 1.535 & -0.927 & -1.839 & 1.533 & -0.942 & -1.819 & 1.559 \\
\hline H18 & 1.004 & -0.401 & 2.059 & 0.999 & -0.399 & 2.057 & 0.997 & -0.397 & 2.063 \\
\hline H19 & -0.176 & 1.993 & 1.308 & -0.177 & 1.991 & 1.305 & -0.127 & 2.015 & 1.316 \\
\hline $\mathrm{H} 2 \mathrm{O}$ & -0.181 & 1.974 & -1.327 & -0.176 & 1.972 & -1.329 & -0.124 & 2.002 & -1.333 \\
\hline $\mathrm{H} 21$ & 2.998 & -0.084 & 0.014 & 3.002 & -0.085 & 0.013 & 3.036 & -0.165 & 0.011 \\
\hline $\mathrm{H} 22$ & 2.764 & -1.619 & 0.899 & 2.766 & -1.612 & 0.899 & 2.742 & -1.683 & 0.895 \\
\hline $\mathrm{H} 23$ & 2.769 & -1.620 & -0.868 & 2.772 & -1.619 & -0.862 & 2.747 & -1.688 & -0.865 \\
\hline $\mathrm{H} 24$ & -3.026 & 0.769 & 0.668 & -3.031 & 0.780 & 0.663 & -3.102 & 0.742 & 0.664 \\
\hline
\end{tabular}




\section{REFERENCES}

1 Fodor, G.; Dharanipragada, R. Tropane Alkaloids, Nat. Prod. Rep., a. 1994, 11, 443-450; b. 1993, 10, 199-206; c. 1991, 8, 603-612; d. 1990, 7, 539-548 for reviews of tropanes and related compounds

2 Jirschitzka, J.; Dolke, F.; D'Auria, J. C. Increasing the pace of new discoveries in tropane alkaloid biosynthesis, Adv. Bot. Res. (2013), 68, 39-72

3 Grynkiewicz, G.; Gadzikowska, M. Tropane alkaloids as medicinally useful natural products and their synthetic derivatives as new drugs, Pharmacol. Rep. (2008), 60, 439-463

4 de Simone, R.; Margarucci, L.; de Feo, V. Tropane alkaloids: an overview, PhOL (Pharmacologyonline) (2008), 1, News Letters, 70-89

5 Fodor, G. Tropane alkaloids, Sainsbury, M. (ed.) Rodd's Chemistry of Carbon Compounds (2nd ed.) (1997), 4(Pt. B), 251-276

6 D. E. Justice; J. R. Malpass, Exo- and Endo- 6-Hydroxy- and 6,7-Epoxytropanes; Total Synthesis of Scopine, Pseudoscopine, and Nor- Derivatives, Tetrahedron Lett. 1995, 36, 4689-4692

7 P. Écija, E. J. Cocinero, A. Lesarri, F. J. Basterretxea, J. A. Fernández, and Fernando Castaño, The Distorted Tropane of Scopoline, ChemPhysChem 2013, 14, 1830-1835

8 Cocinero, E. J.; Lesarri, A.; Ecija, P.; Grabow, J.-U.; Fernandez, J. A.; Castano, F., NMethyl stereochemistry in tropinone: the conformational flexibility of the tropane motif, Phys. Chem. Chem. Phys. (2010), 12, 6076-6083

9 Ecija, P.; Vallejo-Lopez, M.; Evangelisti, L.; Fernandez, J. A.; Lesarri, A.; Caminati, W.; Cocinero, E. J. , O-H $\cdots \mathrm{N}$ and C-H $\cdots$ O Hydrogen Bonds Control Hydration of Pivotal Tropane Alkaloids: Tropinone $\cdots \mathrm{H} 2 \mathrm{O}$ Complex, ChemPhysChem (2014), 15, 918-923

10 J. Balle, W. H. Flygare, Rev. Sci. Instrum. 1981, 52, 33

11 E. J. Cocinero, A. Lesarri, P. Écija, J.-U. Grabow, J. A. Fernández, F. Castaño, Phys. Chem. Chem. Phys. 2010, 12, 12486-12493

12 Powers, D. E.; Hansen, S. G.; Geusic, M. E.; Pulu, A. C.; Hopkins, J. B.; Dietz, T. G.; Duncan, M. A.; Langridged-Smith, P. R. R.; Smalley, R. E. J. Phys. Chem. 1982, 86, 2556

13 Cocinero, E. J., Lesarri, A. Écija, P., Basterretxea, Francisco J., Grabow, J.-U., Fernández, J. A., Castaño, F., Angew. Chem. Int. Ed. 2011, 50, 1.

14 Gaussian 09, Revision A02, M. J. Frisch, G. W. Trucks, H. B. Schlegel, G. E. Scuseria, M. A. Robb, J. R. Cheeseman, G. Scalmani, V. Barone, B. Mennucci, G. A. Petersson, H. Nakatsuji, M. Caricato, X. Li, H. P. Hratchian, A. F. Izmaylov, J. Bloino, G. Zheng, J. L. Sonnenberg, M. Hada, M. Ehara, K. Toyota, R. Fukuda, J. Hasegawa, M. Ishida, T. Nakajima, Y. Honda, O. Kitao, H. Nakai, T. Vreven, J. A. Montgomery, Jr., J. E. Peralta, F. Ogliaro, M. Bearpark, J. J. Heyd, E. Brothers, K. N. Kudin, V. N. Staroverov, R. Kobayashi, J. Normand, K. Raghavachari, A. Rendell, J. C. Burant, S. S. Iyengar, J. Tomasi, M. Cossi, N. Rega, J. M. Millam, M. Klene, J. E. Knox, J. B. 
Cross, V. Bakken, C. Adamo, J. Jaramillo, R. Gomperts, R. E. Stratmann, O. Yazyev, A. J. Austin, R. Cammi, C. Pomelli, J. W. Ochterski, R. L. Martin, K. Morokuma, V. G. Zakrzewski, G. A. Voth, P. Salvador, J. J. Dannenberg, S. Dapprich, A. D. Daniels, Ö. Farkas, J. B. Foresman, J. V. Ortiz, J. Cioslowski, and D. J. Fox, Gaussian, Inc., Wallingford CT, 2009.

15 The M06 suite of density functionals for main group thermochemistry, thermochemical kinetics, noncovalent interactions, excited states, and transition elements: two new functionals and systematic testing of four M06-class functionals and 12 other functionals By Zhao, Yan; Truhlar, Donald G. From Theoretical Chemistry Accounts (2008), 120(1-3), 215-241

16 W. Gordy and R. L. Cook, Microwave Molecular Spectra, Wiley, New York, 1984

17 Watson, J. K. G. in Vibrational Spectra and Structure, ed. Durig, J. R., Elsevier, Amsterdam, 1977, vol. 6, pp. 1-89

18 Zhao, Y. Truhlar, D. G. Acc. Chem. Res. 2008, 41, 157-167

19 a) R. C. Woods, J. Mol. Spectrosc. 1966, 21, 4-24.

b) R. C. Woods, J. Mol. Spectrosc. 1967, 22, 49-59.

c) J. M. Vacherand, B. P. Van Eijck, J. Burie, J. Demaison, J. Mol. Spectrosc. 1986, 118, 355-362; I. Kleiner, J. Mol. Spectrosc. 2010, 260, 1-18 